\title{
Retrospective space-time cluster analysis of whooping cough re-emergence in Barcelona, Spain, 2000-2011
}

\author{
Rubén Solano ${ }^{1,2,3,4}$, Diana Gómez-Barroso ${ }^{3,4}$, Fernando Simón $^{2,3}$, Sarah Lafuente ${ }^{1}$, Pere Simón ${ }^{1}$, \\ Cristina Rius ${ }^{1}$, Pilar Gorrindo ${ }^{1}$, Diana Toledo ${ }^{4,1}$, Joan A. Caylà ${ }^{1,4}$ \\ ${ }^{1}$ Epidemiology Department, Barcelona Public Health Agency, Barcelona, Spain; ${ }^{2}$ Field Epidemiology Training \\ Programme, Carlos III Health Institute, Madrid, Spain; ${ }^{3}$ National Centre for Epidemiology, Carlos III Health \\ Institute, Madrid, Spain; ${ }^{4}$ CIBER Epidemiology and Public Health (CIBERESP), Carlos III Health Institute, \\ Madrid, Spain
}

\begin{abstract}
A retrospective, space-time study of whooping cough cases reported to the Public Health Agency of Barcelona, Spain between the years 2000 and 2011 is presented. It is based on 633 individual whooping cough cases and the 2006 population census from the Spanish National Statistics Institute, stratified by age and sex at the census tract level. Cluster identification was attempted using space-time scan statistic assuming a Poisson distribution and restricting temporal extent to 7 days and spatial distance to $500 \mathrm{~m}$. Statistical calculations were performed with Stata 11 and SatScan and mapping was performed with ArcGis 10.0. Only clusters showing statistical significance $(\mathrm{P}<0.05)$ were mapped. The most likely cluster identified included five census tracts located in three neighbourhoods in central Barcelona during the week from 17 to 23 August 2011. This cluster included five cases compared with the expected level of 0.0021 (relative risk $=2436, \mathrm{P}<0.001$ ). In addition, 11 secondary significant space-time clusters were detected with secondary clusters occurring at different times and localizations. Spatial statistics is felt to be useful by complementing epidemiological surveillance systems through visualizing excess in the number of cases in space and time and thus increase the possibility of identifying outbreaks not reported by the surveillance system.
\end{abstract}

Keywords: space-time analysis, whooping cough, Spain.

\section{Introduction}

Whooping cough remains a public health problem worldwide, clearly reflected in the increasing number of cases reported to the World Health Organization (WHO) in 2001. Western Pacific, Southeast Asia and Africa are the regions with the largest overall number of cases (Tan et al., 2005). This situation has resulted in the creation of the Global Pertussis Initiative (GPI), an expert forum aiming to raise awareness about whooping cough and recommend effective vaccination strategies geared towards the control of the disease (Guiso et al., 2011a). The GPI initiative involves representatives from 21 countries in Africa, the Middle East, Southeast Asia as well as Central and Eastern Europe, (Guiso, 2009; Beltran et al., 2011; Guiso et al., 2011a). In the period 1998-2002, in 12 out of 16 European countries with whooping cough surveillance systems,

\footnotetext{
Corresponding author:

Rubén Solano

Epidemiology Department

Barcelona Public Health Agency, Barcelona, Spain

Tel. +34 93 551-3666. Fax: +34 93 217-3197

E-mail: rsolano@aspb.cat; ruben.solano@gencat.cat
}

the surveillance systems comprised the general population. In 10 countries (Austria, Denmark, France, Germany, Greece, Norway, Portugal, Spain, Sweden and the Netherlands), they were based on the WHO case definition of the disease. Eleven countries applied laboratory tests, and eight of them used the polymerase chain reaction (PCR) for case confirmation (Tozzi et al., 2007). Hospitalization rates in children up to 1 year of age ranged between $33.1 \%$ and $100 \%$, the highest numbers reported from France $(76.1 \%)$; United Kingdom, except Scotland (68.6\%) and Portugal $(53.2 \%)$ (Tozzi et al., 2007). The case fatality rate in this age group ranged between 0 and $2.1 \%$ of the total population, the latter in Portugal (Tozzi et al., 2007).

The number of cases in England and Wales reached 1,781 by late May 2012, compared with a total of 1,118 cases in 2011. In Sweden, a total of 4,992 cases were reported for the period 2002-2006 with the annual incidence decreasing from 70 cases per 100,000 inhabitants in 1992 to 40 per 100,000 inhabitants in 2006 (Wymann et al., 2011; Kmietowicz, 2012). In the late 1970s and early 1980s, Spain and Italy reported an incidence rate between 10 and 100 cases per 100,000 inhabitants (Galazka, 1992). In 
Germany, the annual number of pertussis infections could have exceeded 10,000 in that period, thus producing an incidence rate above 20 cases per 100,000 inhabitants (Galazka, 1992). In Russia, the incidence rate during 1988-1991 ranged between 17 and 24 per 100,000 inhabitants (Galazka, 1992).

Vaccination coverage in Spain increased progressively according to the National Centre for Epidemiology 1 (the Carlos III Health Institute, Madrid) and remained above 90\% since 1996 and over 95\% since 2011. In that year, the national coverage was $97.0 \%$ for the primary series of vaccination (three doses in children $<1$ year of age) and $94.1 \%$ for receiving a booster dose in the second year of life. Since 1996, incidence rates have been on a downward trend but outbreaks are still being reported among children and adolescents with rates between $21.8 \%$ and $26.7 \%$, and an increase in the number of infections in people aged $\geq 35$ years (Torres et al., 2011). The vaccines used were either from GlaxoSmithKline Biologicals or Sanofi-Pasteur-MSD.

Cluster detection is a technique of great interest to epidemiologists and has been used for decades for effective disease management. During the last decade, space-time cluster detection in the health field has been particularly strong, e.g. for the evaluation of infectious diseases such as tuberculosis (Nunes, 2007), malaria (Magalhaes and Clements, 2011), West Nile virus (Winters et al., 2010), schistosomiasis (Clements et al., 2009), H5N1 avian flu (Souris et al., 2010) and hepatitis A (Gomez-Barroso et al., 2011), among others. Detection methods for space-time clusters refer to the identification of a higher density of event occurrences in certain places at certain times, a dynamic technique essential for the determination of when, where and to what degree a disease is present (Galazka, 1992; Garcia-Corbeira et al., 2000; Dominguez et al., 2001; ez-Domingo et al., 2004; Dempsey et al., 2009). The aim of this study was to identify space-time whooping cough clusters at the census tract level during the period 2000-2011 in Barcelona.

\section{Materials and methods}

\section{Study area and data source}

The study covered the Spanish city of Barcelona, which is the capital of the Catalonia Autonomous Community as well as the Barcelona Province. It is

\footnotetext{
${ }^{1}$ Information statement of the epidemiological situation for pertussis in Spain dated 19 September 2012.
}

located on the Mediterranean coast of the Iberian Peninsula and has a population of 1,615,448 inhabitants (2011) and is the second largest city in Spain. According to the Barcelona City Council Statistics Department, the city consists of 10 municipal districts, 73 neighbourhoods and 1,061 census tracts. For this study we used the individual whooping cough cases reported to the Public Health Agency of Barcelona (ASPB) in the period of 2000-2011 and we used the 2006 population census from the National Statistics Institute (INE), stratified by age and sex at the census tract level.

\section{Space-time analysis}

We analysed potential spatio-temporal clusters using the space-time estimator scan statistic developed by Kulldorff et al. (1997) assuming a Poisson distribution and adjusting for sex and age. The estimator consisted of an imaginary "cylinder" with the base representing space and the height time. The spatial window was restricted to a maximum radius of $500 \mathrm{~m}$ and the temporal window limited to 7 days. The entire study area was swept with the base centred on many possible centroids located throughout the territory. During this investigation, the centroid radius size varied from 0 to $500 \mathrm{~m}$ and the height from 0 to 7 days generating an infinite number of cylindrical windows of different sizes and shapes that intersected each other. In this way the entire study territory was eventually covered with each cylinder reflecting a possible cluster.

We tested the hypothesis that the risk was constant in space and time (the null hypothesis) with the hypothesis that the risk was different inside and outside for at least one cylinder (the alternative hypotheses). We recorded the number of observed cases inside and outside for each cylinder along with the number of expected cases according to the model, thus reflecting the population at risk or relative risk (RR). Seen in this way, the cluster with the highest likelihood is the most probable one (Kulldorff et al., 1997). We used the formula below to calculate the likelihood within each circle:

$$
\left(\frac{c}{E[c]}\right)^{c}\left(\frac{C-c}{C-E[c]}\right)^{C-c} I()
$$

(equation 1)

where $C$ is the total case number of cases, $c$ the observed cases within the window and $E[c]$ the adjusted covariate of the expected cases within each window under the null hypothesis. $I()$ is an indicator function that equals 1 when the window has more 
cases than expected under the null hypothesis; otherwise it equals 0 .

We evaluated the observed increase in cases over the expected number with Monte Carlo test simulations (999 iterations) using a 95\% confidence interval. The statistical analysis was performed by SaTScan and Stata version 11 (Kulldorff and Nagarwalla, 1995; Kulldorff et al., 2007; Commenges and Liquet, 2008; Cook et al., 2010). Maps were made with ArcGis, version 10.0 (ESRI; Redlands, USA).

\section{Results}

\section{Cases reported}

In the period under review, 633 whooping cough cases were reported together with individual epidemiological information to the ASPB. Of these, $89.6 \%$ were under 14 years of age; especially many were children 1 year old $(59.8 \%)$ and there was a peak in the children 0-4 year old. Fig. 1 shows the annual case reporting rate by municipal district. In districts with lower number of cases, the temporal pattern appeared stable. From 2007 and up to 2011, all municipalities in Barcelona reported cases of pertussis. The annual distribution of cases by age group (Fig. 2) shows that the highest incidence occurred in the 0-4 age group in each study year. Between 2004 and 2011 there was an increase in the number of cases in all age groups.

\section{Cluster identification}

During the study period, we detected 12 significant clusters, which are shown in Fig. 3 and Table 1. The most likely cluster (red in Fig. 3) occurred in the week of 17-23 August 2011 and comprises five census tracts located in three neighbourhoods of central Barcelona (Vila de Gàcia, Sagrada Família and La Salut). It included five cases whereas 0.0021 cases were expected $(\mathrm{RR}=2,436)$. As far as we know, it was not officially declared an outbreak.

Secondary clusters (blue on the map in Fig. 3) were located at Sant Antoni and Sagrada Família neighbourhoods (from the l'Eixample municipal district); El Baix Guinardó neighbourhood (from the HortaGuinardó municipal district); El Coll, Camp d'en Grassot and Gràcia Nova neighbourhoods (from the Gràcia district); El Turó de la Peira neighbourhood (from the Nou Barris district); and Sants, Sants-Badal and Hostafrancs neighbourhoods (from the SantMontjuiic district). Secondary cluster number 4 was geographically larger. It included 25 census tracts and

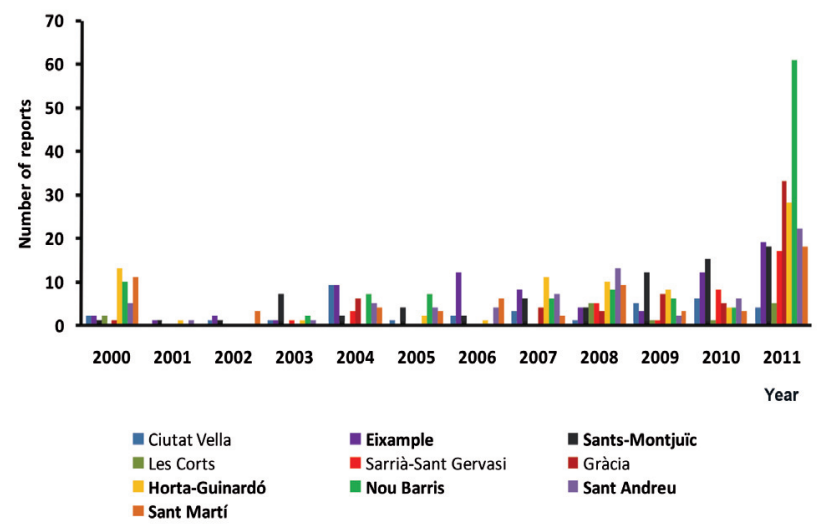

Fig. 1. Annual number of reports by municipal district in Barcelona-Spain, 2000-2011. Data sources: Epidemiology Department, Barcelona Public Health Agency and Barcelona City Council Statistics Department, Spain.

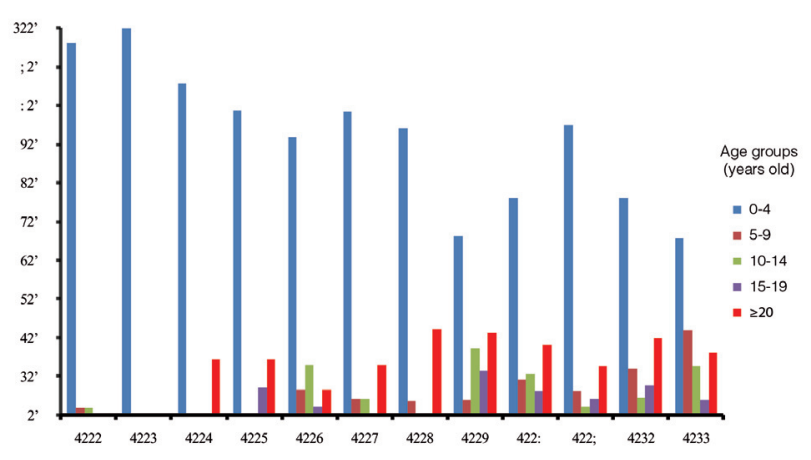

Fig. 2. Annual distribution of cases by age groups in Barcelona between, 2000 and 2011. Data sources: Epidemiology Department, Barcelona Public Health Agency, Spain.

was declared an outbreak. Overall, we observed 11 statistically significant, secondary clusters, most of which appeared during the summer months.

\section{Discussion}

This study represents the first analysis of the spacetime whooping cough clusters in the city of Barcelona. Although there are studies related to exemption requirements from immunization in school children and their associations with geographic clustering of infection (Crespo et al., 2011), no other study in Spain has focused on the detection of whooping cough clusters.

Between 2000 and 2011, the official rate of reported whooping cough cases from the districts of L'Exaimple, Sants-Montjuï, Sant Andreu, HortaGuinardó, Sant Martí and Nou Barris was higher than than that of any other district in Barcelona. Although a clear association between this result and the epi- 


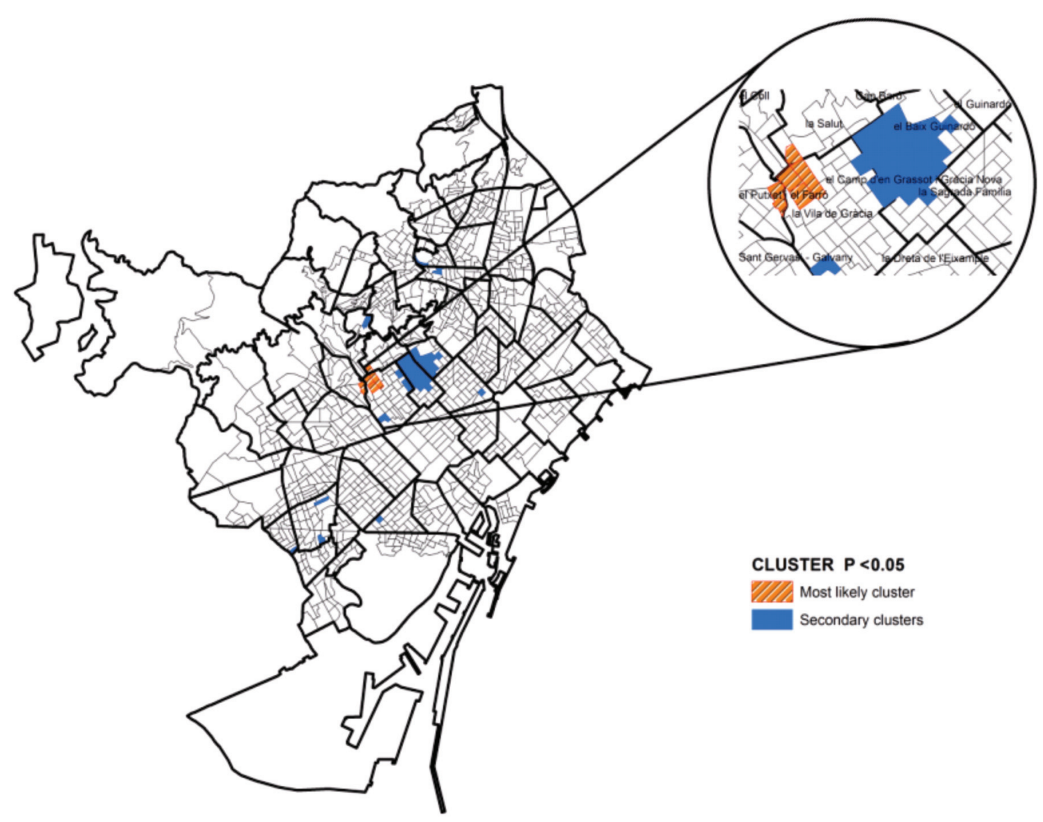

Fig. 3. Space-time distribution of clusters of whooping cough in Barcelona, Spain between 2000 and 2011. Data sources: Epidemiology Department, Barcelona Public Health Agency, Spain.

demiological surveillance in each district cannot be established, we note that these parts of the city are among the most populated ones.

The main cluster included five census tracts in central Barcelona and comprised some of the most densely populated neighbourhoods. According to information from the Spanish Statistical Institute of Catalonia, La Vila de Gràcia (belonging to the Gràcia municipal district) the proportion of children aged $<4$ years in 2011 was $10.5 \%$, that of the $15-24$ age group $27.3 \%$ and among adults $62.2 \%$. More than half the popula- tion $(53 \%)$ had been born and grown up in this area with a density of 38.4 inhabitants $/ \mathrm{km}^{2}$ and a weekly per capita family income of $€ 102.8$. La Salut (also belonging to Gràcia municipal district) had a similar pattern: $11.5 \%$ children, $31.6 \%$ of the $15-24$ age group and $56.9 \%$ adults. The indigenous part of the population was the same $(53 \%)$ with a density of 20.5 inhabitants $/ \mathrm{km}^{2}$ and a per capita family income of $€$ 111.7 per week. In 2010, in neighbourhoods Sagrada Família and El Poblet in the L'Eixample municipal district there were $10.2 \%$ children, $30.5 \% \quad 15-24$ year

Table 1. Space-time distribution of whooping cough clusters in Barcelona, Spain, 2000-2011.

\begin{tabular}{lccccc}
\hline Cluster & Time period & Cases observed & Cases expected & P-value & NCTs* concerned \\
\hline 1 & $17 / 08 / 2011-23 / 08 / 2011$ & 5 & 0.0021 & $<0.001$ & 5 \\
2 & $30 / 03 / 2011-05 / 04 / 2011$ & 3 & 0.0005 & $<0.001$ & 1 \\
3 & $07 / 11 / 2007-13 / 11 / 2007$ & 3 & 0.0006 & 0.001 & 1 \\
4 & $29 / 06 / 2011-05 / 07 / 2011$ & 4 & 0.0110 & 0.005 & 25 \\
5 & $22 / 09 / 2010-28 / 09 / 2010$ & 3 & 0.0017 & 0.006 & 1 \\
6 & $06 / 08 / 2003-12 / 08 / 2003$ & 3 & 0.0034 & 0.018 & 1 \\
7 & $15 / 06 / 2011-21 / 06 / 2011$ & 2 & 0.0005 & 0.019 & 1 \\
8 & $11 / 02 / 2004-17 / 02 / 2004$ & 2 & 0.0005 & 0.025 & 1 \\
9 & $14 / 07 / 2010-20 / 07 / 2010$ & 2 & 0.0005 & 0.030 & 1 \\
10 & $28 / 01 / 2004-03 / 02 / 2004$ & 2 & 0.0006 & 0.040 & 1 \\
11 & $26 / 09 / 2007-02 / 10 / 2007$ & 2 & 0.0006 & 0.044 & 1 \\
\end{tabular}

*Number of cencus tracts. Data sources: Epidemiology Department, Barcelona Public Health Agency and National Statistics Institute, Spain. 
olds and $59.3 \%$ adults, the indigenous population making up $51.6 \%$ of the total population. The density was 49.4 inhabitants $/ \mathrm{km}^{2}$ with a per capita family income of $€ 97.3$ per week. Although the role played by population density, migration and income was not investigated, it is quite clear that low income and living in close quarters contribute to increased transmission and susceptibility not only to pertussis but also other infectious diseases.

Whooping cough is characteristically underreported and is probably under-diagnosed in daily clinical practice and at primary-care centres because it is believed to have been more or less eliminated. It is therefore necessary to intensify work on strengthening the existing reporting system with respect to data quality. This could be achieved by implementing a sentinel network of paediatricians and primary-care physicians that could act on the underreporting issue. It is estimated that between $47 \%$ and $80 \%$ among adults with whooping cough are misdiagnosed due to presenting with only mild clinical symptoms making it necessary to establish a proper differential diagnosis with other upper respiratory tract diseases and other sometimes forgotten, clinically similar infections (Moretti et al., 2000; Guinto-Ocampo et al., 2008; Dempsey et al., 2009). Serology would contribute to a correct diagnosis. For example, studies conducted in other parts of Spain on vaccination coverage and outbreaks among children, adolescents and adults, specifically in Catalonia and Valencia, have approached the problem by assessing the prevalence of $\operatorname{IgG}$ antibodies against pertussis toxin to estimate the annual burden of whooping cough hospitalizations (Montiano Jorge et al., 1992; Diez-Domingo et al., 2004).

We found the highest incidence rates in children aged $<4$ years (Fig. 2). A similar pattern is seen in several countries in central and Eastern Europe, where the high vaccination coverage in children has made the distribution of cases appear to have been moved from young children to adolescents and adults. The most likely explanation for this is loss of immunity in the older age groups, who then transmit the infection. Supporting facts are common misdiagnosis of coughing, particularly in older patients and although acellular vaccines are available in the private market, follow-up booster vaccination after childhood is not mandatory (Lutsar et al., 2009; Guiso et al., 2011a). Indeed, our study observed that there was a relatively high rate of infection among adults with the proportion of adults (here presented as those older than 20 year) generally next in numbers after the $0-4$ age group (Fig. 2). This fact is important for the possible design of more effective vac- cination schedules and quantification of the dependent relationship between age and number of infected cases reported. This strategy has already been put in place in some other European countries, e.g. the Netherlands and the Czech Republic, where a high rate of infection in older people has been observed (de Melker et al., 2006; Guiso et al., 2011a, 2011b).

The method introduced by Kulldorff et al. (2007), which is commonly used for spatio-temporal cluster analysis, has several advantages: it conforms to the population density and confounding variables such as age and sex, and there is no pre-selection bias because groups are searched without prior assumptions about their location period, size or time. This statistical method takes into account multiple testing; allowing us to obtain a single P-value, and it locates and specifies the occurrence of the cluster dates. Cases from clusters 1 and 4 were reported in 2011 by the end of May, and although cluster 4 was reported as outbreak, there was no evidence that the most likely cluster was as well. The scan method is able to detect as clusters those outbreaks that have been notified and also identify other clusters that have not been declared and should be investigated.

This technique has been applied to other diseases not analysed in this study. For example, Nunes (2007) used these techniques to analyse the incidence of tuberculosis in Portugal, while Odoi et al. (2004) investigated timespace cluster of giardiasis in Canada. Sugumaran et al. (2009) conducted a space-time study for the West Nile Virus using this methodology and Gómez-Barroso et al. (2012) investigated a time-space cluster of hepatitis A in Spain using scan method. These methods may also have the potential to detect clusters of new or emerging disease. However, not all clusters detected using statistical analysis will represent real outbreaks and further epidemiological investigation is required to determine whether these statistical clusters represent real outbreaks (Hyder et al., 2011).

\section{Conclusion}

Communicable diseases, such as whooping cough, often spread in unforeseen ways and cannot always be controlled by reviewing control measures and preventive immunization programmes. Space-time exploration enhances effectiveness by swiftly directing control measures to specific areas, thus contributing to the assessment of health programmes related to the event. We propose implementation of this analysis methodology for future studies of other diseases and its routine use in epidemiological surveillance systems. 


\section{Acknowledgements}

The authors wish to express sincere thanks to Drs. Juan de Mata Donado Campos, Maria Victoria de Aragón y Esquivas, Alicia Barrasa, Josefa Masa and Pere Godoy for their suggestions. We thank their many fields work nursing involved in the surveys. We also thank the Epidemiology Department of Public Health Agency of Barcelona, for facilitating the surveys and database. The authors also wish to thank two anonymous reviewers for their comments and suggestions which substantially improved the manuscript.

\section{References}

Beltran SS, Cervantes AY, Cherry JD, Conde GC, Gentile A, Gomez Altamirano CM, Hernandez PM, Huerta GG, Macias PM, Martinez AG et al., 2011. Consensus on the clinical and microbiologic diagnosis of Bordetella pertussis, and infection prevention. Expert Group on Pertussis Vaccination. Salud Publica Mex 53, 57-65.

Clements AC, Bosque-Oliva E, Sacko M, Landoure A, Dembele R, Traore M, Coulibaly G, Gabrielli AF, Fenwick A, Brooker S, 2009. A comparative study of the spatial distribution of schistosomiasis in Mali in 1984-1989 and 2004-2006. PLoS Negl Trop Dis 3, 431.

Commenges D, Liquet B, 2008. Asymptotic distribution of score statistics for spatial cluster detection with censored data. Biometrics 64, 1287-1289.

Cook AJ, Li Y, Arterburn D, Tiwari RC, 2010. Spatial cluster detection for weighted outcomes using cumulative geographic residuals. Biometrics 66, 783-792.

Crespo I, Cardenosa N, Godoy P, Carmona G, Sala MR, Barrabeig I, Alvarez J, Minguel S, Camps N, Cayla J et al., 2011. Epidemiology of pertussis in a country with high vaccination coverage. Vaccine 29, 4244-4248.

de Melker HE, Versteegh FG, Schellekens JF, Teunis PF, Kretzschmar M, 2006. The incidence of Bordetella pertussis infections estimated in the population from a combination of serological surveys. J Infect 53, 106-113.

Dempsey AF, Cowan AE, Broder KR, Kretsinger K, Stokley S, Clark SJ, 2009. Diagnosis and testing practices for adolescent pertussis among a national sample of primary care physicians. Prev Med 48, 500-504.

Diez-Domingo J, Ballester A, Baldo JM, Planelles MV, Villarroya JV, Alvarez T, Carmen PM, Calero P, Garces MD, Sorribes I et al., 2004b. Incidence of pertussis in persons < or $=15$ years of age in Valencia, Spain: seroprevalence of antibodies to pertussis toxin (PT) in children, adolescents and adults. J Infect 49, 242-247.

Dominguez A, Vidal J, Plans P, Salleras L, 2001. The seroepidemiology of $B$. pertussis infection in Catalonia, Spain. Epidemiol Infect 126, 205-210.
Galazka A, 1992. Control of pertussis in the world. World Health Stat Q 45, 238-247.

Garcia-Corbeira P, Dal-Re R, Aguilar L, Garcia-de-Lomas J, 2000. Seroepidemiology of Bordetella pertussis infections in the Spanish population: a cross-sectional study. Vaccine 18, 2173-2176.

Gomez-Barroso D, Rodriguez VE, Flores S, Ramis PR, Barrio Fernandez JL, Simon SF, 2009. Space distribution of tuberculosis in Spain by geostatistical methods. Rev Esp Salud Publica 83, 737-744.

Gomez-Barroso D, Varela C, Ramis R, Del Barrio JL, Simon F, 2012. Space-time pattern of hepatitis A in Spain, 1997-2007. Epidemiol Infect 140, 407-416.

Guinto-Ocampo H, Bennett JE, Attia MW, 2008. Predicting pertussis in infants. Pediatr Emerg Care 24, 16-20.

Guiso N, 2009. Bordetella pertussis and pertussis vaccines. Clin Infect Dis, 49,1565-1569.

Guiso N, Liese J, Plotkin S, 2011b. The Global Pertussis Initiative: meeting report from the fourth regional roundtable meeting, France, April 14-15, 2010. Hum Vaccin 7, 481-488.

Guiso N, Wirsing von Konig CH, Forsyth K, Tan T, Plotkin SA, 2011a. The Global Pertussis Initiative: report from a round table meeting to discuss the epidemiology and detection of pertussis, Paris, France, 11-12 January 2010. Vaccine 29, 1115-1121.

Hyder K, Vidal-Diez A, Lawes J, Sayers AR, Milnes A, Hoinville L, Cook AJ, 2011. Use of spatiotemporal analysis of laboratory submission data to identify potential outbreaks of new or emerging diseases in cattle in Great Britain. BMC Vet Res 7, 14.

Kmietowicz Z, 2012. Pertussis cases rise 10-fold among older children and adults in England and Wales. BMJ 345, 5008.

Kulldorff M, Feuer EJ, Miller BA, Freedman LS, 1997. Breast cancer clusters in the northeast United States: a geographic analysis. Am J Epidemiol 146, 161-170.

Kulldorff M, Mostashari F, Duczmal L, Katherine YW, Kleinman K, Platt R, 2007. Multivariate scan statistics for disease surveillance. Stat Med 26, 1824-1833.

Kulldorff M, Nagarwalla N, 1995. Spatial disease clusters: detection and inference. Stat Med 14, 799-810.

Lutsar I, Anca I, Bakir M, Usonis V, Prymula R, Salman N, Grezesiowski P, Greenberg M, 2009. Epidemiological characteristics of pertussis in Estonia, Lithuania, Romania, the Czech Republic, Poland and Turkey-1945 to 2005. Eur J Pediatr 168, 407-415.

Magalhaes RJ, Clements ACA, 2011. Mapping the risk of anaemia in preschool-age children: the contribution of malnutrition, malaria, and helminth infections in West Africa. PLoS Med 8, e1000438.

Montiano Jorge JI, Lazcoz HA, Amiama, P, Mendia GM, Benito FJ, Santiago BM., Pocheville GI, Gutierrez VC, Gaztelurrutia AL, 1992. Pertussis. Study of an epidemic. An Esp Pediatr 37, 
184-186.

Moretti F, Chellini E, Baretti S, Santini MG, Rosati GV, Comodo N, 2000. Estimate of underreporting of infectious diseases through a sentinel network of pediatricinas in the area of local health unit of Florence. Epidemiol Prev 24, 224-227.

Nunes C, 2007. Tuberculosis incidence in Portugal: spatiotemporal clustering. Int J Health Geogr 6, 30.

Odoi A, Martin SW, Michel P, Middleton D, Holt J, Wilson J, 2004. Investigation of clusters of giardiasis using GIS and a spatial scan statistic. Int J Health Geogr 3, 11.

Souris M, Gonzalez JP, Shanmugasundaram J, Corvest V, Kittayapong P, 2010. Retrospective space-time analysis of H5N1 avian influenza emergence in Thailan. Int J Health Geogr 9, 3.

Sugumaran R, Larson SR, Degroote JP, 2009. Spatiotemporal cluster analysis of county-based human West Nile virus incidence in the continental United States. Int J Health Geogr 8, 43.
Tan T, Trindade E, Skowronski D, 2005. Epidemiology of pertussis. Pediatr Infect Dis J 24, 10-18.

Torres J, Godoy P, Artigues A, Codina G, Bach P, Modol I, Duro MA, Trilla C, 2011. Outbreak of whooping cough with a high attack rate in well-vaccinated children and adolescents. Enferm Infecc Microbiol Clin 29, 564-567.

Tozzi AE, Pandolfi E, Celentano LP, Massari M, Salmaso S, Ciofi degli Atti ML, 2007. Comparison of pertussis surveillance systems in Europe. Vaccine 25, 291-297.

Winters AM, Eisen RJ, Delorey MJ, Fischer M, Nasci RS, Zielinski-Gutierrez E, Moore CG, Pape WJ, Eisen L, 2010. Spatial risk assessments based on vector-borne disease epidemiologic data: importance of scale for West Nile virus disease in Colorado. Am J Trop Med Hyg 82, 945-953.

Wymann MN, Richard JL, Vidondo B, Heininger U, 2011. Prospective pertussis surveillance in Switzerland, 1991-2006. Vaccine 29, 2058-2065. 